\title{
Comparative study of irradiation effects in graphite and graphene induced by swift heavy ions and highly charged ions
}

\author{
J. Zeng ${ }^{\mathrm{a}, *}$, J. Liu ${ }^{\mathrm{a}, *}$ H.J. Yao ${ }^{\mathrm{a}}$, P.F. Zhai ${ }^{\mathrm{a}}$, S.X. Zhang ${ }^{\mathrm{a}, \mathrm{b}}$, H. Guo ${ }^{\mathrm{a}, \mathrm{b}}$, P.P Hu ${ }^{\mathrm{a}, \mathrm{b}}$, J.L. Duan ${ }^{\mathrm{a}}$, D. Mo ${ }^{\mathrm{a}}$, M.D. Hou ${ }^{\mathrm{a}}$, \\ and Y.M. Sun ${ }^{\mathrm{a}}$ \\ ${ }^{a}$ Institute of Modern Physics, Chinese Academy of Sciences (CAS), Lanzhou 730000, PR China \\ ${ }^{b}$ University of Chinese Academy of Sciences, Beijing 100049, PR China
}

Abstract

Highly oriented pyrolytic graphite (HOPG) and monolayer graphene were irradiated by swift heavy ions (SHI, $479 \mathrm{MeV}^{86} \mathrm{Kr}$ and $250 \mathrm{MeV}^{112} \mathrm{Sn}$ ) and highly charged ions (HCI, $4 \mathrm{MeV}^{86} \mathrm{Kr}^{19+}$ ). The irradiation effects caused by different types of irradiation were investigated by Raman spectroscopy. It was found that the intensity ratio of D peak to G peak $\left(I_{\mathrm{D}} / I_{\mathrm{G}}\right)$ in the case of HCI was higher than that of SHI for the same ion fluence in HOPG. The larger $I_{\mathrm{D}} / I_{\mathrm{G}}$ indicates that synergistic effects of kinetic and potential energies of medium energy HCI has to be considered during the energy deposition process. A turning point was detected during the evolution process of $I_{\mathrm{D}} / I_{\mathrm{G}}$ with fluence obtained from SHI and HCI impacted graphene, while such turning point was absent in the case of HOPG. The Lucchese's phenomenological model was modified and the experimental data of $I_{\mathrm{D}} / I_{\mathrm{G}}$ vs. fluence for HOPG and graphene was completely following the modified model. According to this model, energetic ions induced both structurally disordered and activated regions in graphene. The competing mechanism of these two regions resulted in three different trends of the $I_{\mathrm{D}} / I_{\mathrm{G}}$ variation in the case of graphene whereas in HOPG, such mechanism was not observed.

\footnotetext{
* Corresponding author. Tel: +86 0931 4969331. E-mail: zengjian@impcas.ac.cn (Jian Zeng).

* Corresponding author. Tel: +86 0931 4969334. E-mail: j.liu@impcas.ac.cn (Jie Liu).
} 
Different aspects of ion beam irradiation induced damage in the materials have been the subject of interest for research. Swift heavy ions (SHI) and highly charged ions (HCI) are two different kinds of sources used in ion beam irradiation experiments. The term "swift" means the velocity of the ions is comparable to the Bohr velocity of the electron [1] and "heavy ions" indicates that their mass number is greater than 4. SHI transfer their energy to the materials when injected into a target. The stopping process of the ions is dominated by inelastic collisions with the target electrons, thereby causing excitation and ionization processes (electronic stopping). Each individual projectile induces a long, straight nanometric damage trail consisting of amorphous or otherwise modified material, called latent ion track. Elastic collisions (nuclear stopping) play a minor role, except at the very end of the ion trajectory. A highly charged ion is an unique particle with a large potential energy Ep, which is stored due to the ionization of many or most of the bound electrons from a neutral atom [2]. HCI can induce emission of multiple electrons from a target surface thus modifying the local electronic states of the material surface significantly. The underlying mechanism of this effect is thought to be distinctively different from the low energy ion irradiation effects, where only the kinetic energy of the primary particle is transferred to the target atoms. The main differences between the two types of ions are the ion energy and energy deposition in the target. SHI are $\mathrm{GeV}$ range). SHI are characterized by the fact that electronic stopping dominates over nuclear stopping process $[1,2]$. HCI are mainly characterized by their low velocities and high potential energies [2]. Medium energy HCI, e.g. $4 \mathrm{MeV} \mathrm{Kr}^{19+}$ used in this work, can be considered as an intermediate regime between the SHI and low energy HCI. The synergistic effects of kinetic and potential energies of medium energy HCI need to be considered during the energy deposition process in the materials [3].

In many solids, hillocks are induced by SHI at the surface with a radius that depends on the electronic energy loss [4-8]. Similar surface nanostructures were also detected on HCI impacted materials [9-12]. The similarity among the nanostructures indicates that a common mechanism related to the energy transfer process is involved in both irradiation types. Comparative study of the 
irradition effects caused by SHI and HCI provides an improved understanding on the energy transfer mechanism and related irradiation effects.

Highly oriented pyrolytic graphite (HOPG) could be used as a moderating and shielding material for nuclear reactors. In order to have a better understanding about the irradiation effects caused by neutrons in HOPG, SHI irradiation is used to simulate the radiation environment in a nuclear reactors. The tracks induced by SHI in HOPG have been systematically invistigated for many years $[13,14]$. Graphene, composed of a hexagonal two-dimensional network of carbon atoms $[15$, 16], can be considered as the parental compound for the carbon allotropes of other dimensionalities. Investigation of irradiation effects on graphene paves the way to better understand ion-matter interactions in other carborn allotropes.

Most of the investigations focus on low- and medium-energy electron-beams $[17,18]$ and ion-beams (with energies in the range $\mathrm{eV} / \mathrm{u}$ to $\mathrm{keV} / \mathrm{u}$ ) [19-21] irradiation effects on graphene. A. Jorio et al.[22] used Raman scattering to study the effect of low energy $(90 \mathrm{eV}) \mathrm{Ar}^{+}$ion bombardment in N-layers graphene. They provided a spectroscopic technique to study the penetration of low energy $\mathrm{Ar}^{+}$ions in $\mathrm{AB}$ Bernal stacked graphite and post irradiation affect on the graphene sheets. E. H. Martins Ferreira et al. [23] reported the irradiation results of different layers of graphene and graphite bombarded by low-energy SHI ions $\left(90 \mathrm{eV} \mathrm{Ar}^{+}\right)$. The evolution of the main Raman bands of monolayer, bilayer, trilayer graphene, and graphite was modeled. M.M. Lucchese et al. [21] proposed a phenomenological model to quantify the density of defects in graphene impacted by $90 \mathrm{eV} \mathrm{Ar}^{+}$ions. Later, Ronaldo Giro et al. [24] extended the phenomenological model to quantifying defects in N-layer graphene using Raman spectroscopy. Up to now, only a few groups have investigated the irradiation effect of medium engery HCI on graphene. Moreover, graphene can be viewed as a single layer of HOPG. Since SHI traverse deeply into a target, inducing a long latent track, and HCI produce structural changes on the surface due to potential energy deposition, therefore, both HOPG and monolayered graphene are two ideal targets for comparison of irradiation effects induced by highly charged ions and swift heavy ions. Comparative study of irradiation effects in graphite and graphene induced by swift heavy ions and highly charged ions also paves a way to understand different reactions of $2 \mathrm{D}$ and $3 \mathrm{D}$ materials to the impacting ions.

In this article, we report the results of irradiation experiments on HOPG and graphene samples using HCI $\left({ }^{86} \mathrm{Kr}^{19+}\right)$ and SHI $\left({ }^{86} \mathrm{Kr},{ }^{112} \mathrm{Sn}\right)$ with energies of $4 \mathrm{MeV}, 479 \mathrm{MeV}$ and $250 \mathrm{MeV}$, 
1 respectivly. The pristine and irradiated samples were inverstigated using Raman spectroscopy. The observed irradiation effects are discussed in terms of similarities and differences of HCI and SHI ion

3 projectiles in graphene and HOPG target.

\section{Experiment}

HOPG (Advanced Ceramics Co., Grade ZYB) were cut into pieces of $0.7 \times 0.7 \times 0.1 \mathrm{~cm}^{3}$ and adhered to a copper sample holder. Monolayer graphene samples were successfully fabricated using the micromechanical cleaving technique suggested by A.K. Geim and K.S. Noeoselov et al. [25, 26] Silicon ( $\mathrm{Si}$ ) wafers with a $300 \mathrm{~nm} \mathrm{SiO}_{2}$ capping layer were used as the substrate for the graphene. Letters and numbers were marked on the top surface of $\mathrm{SiO}_{2} / \mathrm{Si}$ assisted with electron beam lithography. The distance between the two successive marks was fixed to $250 \mu \mathrm{m}$. These marks can help in finding the graphene layers after irradiation. No further cleaning or processing of graphene samples on $\mathrm{SiO}_{2} / \mathrm{Si}$ substrate was carried out in order to keep its pristine properties. Optical microscope was used to map the graphene sheets and roughly identify graphene layers with different thicknesses. The thinner graphene sheets were further studied by Raman spectroscopy to verify the presence of monolayer graphene. With the marks on $\mathrm{SiO}_{2} / \mathrm{Si}$ substrate, the confirmed monolayer graphene could be easily detected after irradiation.

\section{Table 1}

Irradiation parameters in HOPG and graphene. $(\mathrm{d} E / d x)_{\mathrm{e}}$ and $(\mathrm{d} E / \mathrm{d} x)_{\mathrm{n}}$ denote for the electronic and nuclear energy losses, respectively, as calculated with the SRIM2013 code.

\begin{tabular}{cccccccc}
\hline $\begin{array}{c}\text { Ion } \\
\text { type }\end{array}$ & Ions & $\begin{array}{c}\text { Energy } \\
(\mathbf{M e V})\end{array}$ & $\begin{array}{c}\text { Relative } \\
\text { velocity } \\
(\boldsymbol{\beta}=\mathbf{v} / \mathbf{c})\end{array}$ & $\begin{array}{c}(\mathbf{d} E / \mathbf{d} \boldsymbol{x})_{\mathbf{e}} \\
(\mathbf{k e V} / \mathbf{n m})\end{array}$ & $\begin{array}{c}(\mathbf{d} E / \mathbf{d} \boldsymbol{x})_{\mathbf{n}} \\
\left(\times 10^{-2}\right) \\
(\mathbf{k e V} / \mathbf{n m})\end{array}$ & $\begin{array}{c}\text { Range in } \\
\mathbf{H O P G}(\boldsymbol{\mu m})\end{array}$ & $\begin{array}{c}\text { ion fluence } \\
\left(\mathbf{c m}^{-2}\right)\end{array}$ \\
\hline \multirow{2}{*}{$\mathrm{SHI}$} & ${ }^{112} \mathrm{Sn}$ & 250 & 0.07 & 16.5 & 3.2 & 22.1 & $1.0 \times 10^{11}-1.0 \times 10^{14}$ \\
& ${ }^{86} \mathrm{Kr}$ & 479 & 0.11 & 9.9 & 0.8 & 48.0 & $5.0 \times 10^{11}-3.0 \times 10^{13}$ \\
$\mathrm{HCI}$ & ${ }^{86} \mathrm{Kr}^{19+}$ & 4 & 0.01 & 2.4 & 36 & 1.8 & $1.2 \times 10^{11}-3.1 \times 10^{16}$ \\
\hline
\end{tabular}

Both SHI and HCI were used to irradiate HOPG and monolayer graphene. Details of the

21 irradiation parameters are summarized in Table 1. The irradiation experiments with highly charged

22 ions were carried out at the $320 \mathrm{kV}$ platform for multi-discipline research at Institute of Modern

23 Physics, Chinese Academy of Sciences (IMP, CAS). SHI $\left({ }^{86} \mathrm{Kr}\right.$ and ${ }^{112} \mathrm{Sn}$ ions) with initial kinetic 
energy of $25 \mathrm{MeV} / \mathrm{u}$ and $3.7 \mathrm{MeV} / \mathrm{u}$ were provided by two cyclotrons of the Heavy Ion Research Facility in Lanzhou (HIRFL), IMP, CAS. Irradiation experiments were performed at room temperature in vacuum. The flux of $\mathrm{Sn}$ and $\mathrm{Kr}$ was about $5 \times 10^{8}$ ions $/\left(\mathrm{cm}^{2} \cdot \mathrm{s}\right)$ and $1 \times 10^{8}$ ions $/\left(\mathrm{cm}^{2} \cdot \mathrm{s}\right)$, respectively. The flux of $\mathrm{HCI}\left(\mathrm{Kr}^{19+}\right)$ was about $6 \times 10^{10}$ ions $/\left(\mathrm{cm}^{2} \cdot \mathrm{s}\right)$. A Faraday cylinder and a 3-foil aluminum detector were used in SHI irradiation experiment for calibration of fluence. Two Faraday cylinders were used for HCI fluence calibration. The electronic and nuclear energy losses and penetration depth of the ions in HOPG were calculated using the Stopping and Range of Ions in Matter (SRIM2013) code [27, 28].

A Microscopic confocal Raman spectrometer (LabRAM HR800, Jobin Yvon Co.) was used to characterize HOPG and graphene samples before and after the irradiation. The excitation wavelength of the Raman spectrometer was $532 \mathrm{~nm}$, and the optical skin depth in graphite was approximately 50 $\mathrm{nm}$ [29-31]. The penetration depths of all the particles used in this work are larger than $50 \mathrm{~nm}$ as shown in Table.1. The thickness of HOPG samples are about $0.1 \mathrm{~cm}$. Thus, we can assume that the observed Raman spectra are taken from the damaged volumes of HOPG. Raman shift from 1100 $\mathrm{cm}^{-1}$ to $3500 \mathrm{~cm}^{-1}$ was chosen, as in this regime all Raman peaks of HOPG and graphene could be detected and the unwanted Raman signal from the $\mathrm{SiO}_{2} / \mathrm{Si}$ substrate $\left(\sim 520.7 \mathrm{~cm}^{-1}\right.$ and $\left.\sim 940-976 \mathrm{~cm}^{-1}\right)$ could be avoided.

The irradiated HOPG samples were further investigated using scanning tunneling microscope (STM) with mechanical sheared Pt-Ir tips at room temperature. The scanning area was minimized down to $8 \times 8 \mathrm{~nm}^{2}$ in order to measure the defect morphology accurately.

\section{Results and discussions}

\subsection{Irradiation effects in HOPG}

The Raman spectra of both pristine and irradiated samples by SHI and HCI are shown in Fig. 1a, $1 \mathrm{~b}$ and 1c. During the irradiation of $479 \mathrm{MeV} \mathrm{Kr}$ ions on HOPG, a new peak denoted as D peak $\left(\sim 1338 \mathrm{~cm}^{-1}\right)$ appears in addition to the original $\mathrm{G}$ peak $\left(\sim 1582 \mathrm{~cm}^{-1}\right)$ when the fluence is $5 \times 10^{12}$ ions $/ \mathrm{cm}^{2}$ or higher (insert of Fig. 1a). Two additional peaks, D peak and D' peak $\left(\sim 1622 \mathrm{~cm}^{-1}\right)$, were observed in the Raman spectra when irradiated by $250 \mathrm{MeV}$ Sn (Fig. 1b) and $4 \mathrm{MeV} \mathrm{Kr}{ }^{19+}$ ions (Fig. 1c). The assignment of the peaks is straightforward in the molecular picture of the carbon materials. The $\mathrm{G}$ peak is due to the stretching of all $\mathrm{sp}^{2}$ bonds in both rings and chains. The $\mathrm{D}$ peak is due to the 
1 breathing modes of $\mathrm{sp}^{2}$ bonds in rings that are not Raman active and require defects for their 2 activation [32-34]. The $\mathrm{D}^{\prime}$ peak is defect related and occurs via an intra-valley double resonance 3 process in the presence of defects. The additional $\mathrm{D}$ and $\mathrm{D}^{\prime}$ peaks are the two most prominent 4 features in the Raman spectra of the disordered graphite, and the relative Raman intensities of these 5 two peaks are of the order of $I_{\mathrm{D}} / I_{\mathrm{D}} \approx 10$ [34]. It is therefore easy to understand that the $\mathrm{D}^{\prime}$ peaks 6 present only in Fig. $1 \mathrm{~b}$ and c. The $\mathrm{D}^{\prime}$ peak produced by $479 \mathrm{MeV} \mathrm{Kr}$ ions (Fig. 1a) may appear at 7 much higher fluence. In general, the $\mathrm{D}^{\prime}$ peak is not much studied because it appears just as a small 8 shoulder of the G peak. However, Eckmann et al. [35] did a very interesting work, and they pointed 9 out that $I_{\mathrm{D}} / I_{\mathrm{D}^{\prime}}$ could be used to identify the nature of defects. The maximum value of $I_{\mathrm{D}} / I_{\mathrm{D}^{\prime}}$ is about 13 10 for defects associated with $\mathrm{sp}^{3}$ hybrization. $I_{\mathrm{D}} / I_{\mathrm{D}^{\prime}}$ decreases for vacancy-like defects $(\sim 7)$ and reaches 11 a minimum $(\sim 3.5)$ for boundaries in graphite. The $I_{\mathrm{D}} / I_{\mathrm{D}^{\prime}}$ in Fig. $1 \mathrm{~b}$ and Fig. $1 \mathrm{c}$ is about 4 and 5 , 12 respectively. Which indicates the main type of defects in HOPG irradiated by $250 \mathrm{MeV} \mathrm{Sn}$ ions and 4 $13 \mathrm{MeV} \mathrm{Kr}^{19+}$ ions are the boundaries in graphite.
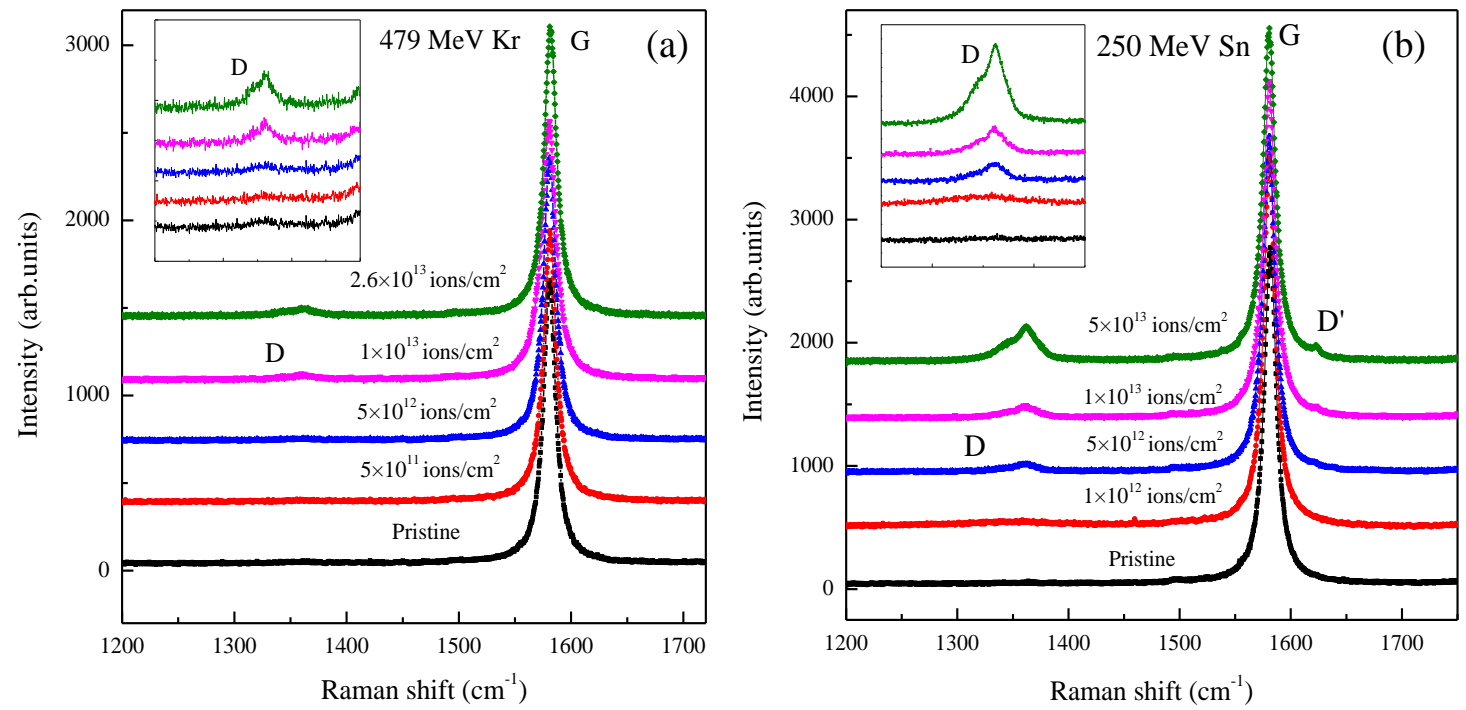


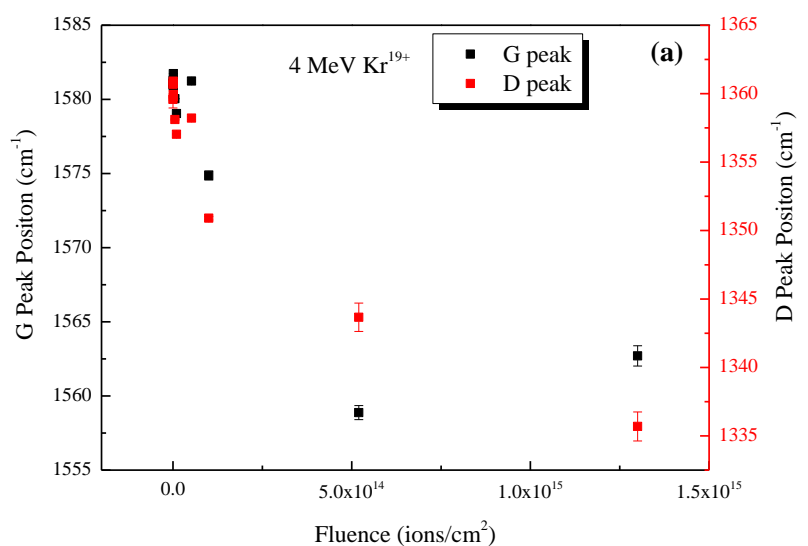
compared to the case of SHI irradiation.

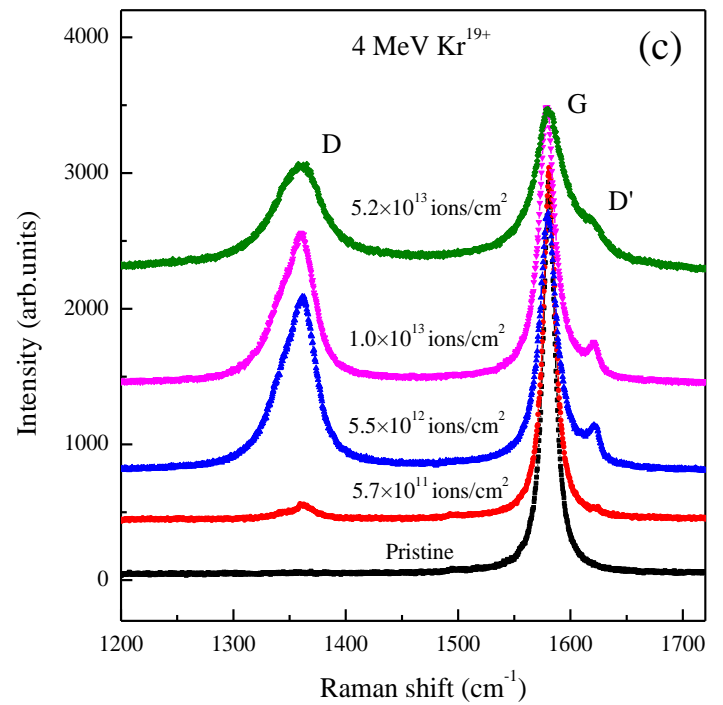

Fig. 1 - Raman spectra of HOPG before and after irradiation at different fluences with (a) $479 \mathrm{MeV} \mathrm{Kr}$ ion ions; (b) 250 MeV Sn ions; (c) $4 \mathrm{MeV} \mathrm{Kr}^{19+}$ ions.

A comparison of the Raman spectra for SHI (Fig. 1a and b) and HCI (Fig. 1c) at similar fluences shows a significant difference in the radiation response with respect to both types of ion beams. At the same fluence, the intensity of D peak in the case of HCI irradiation is much larger as
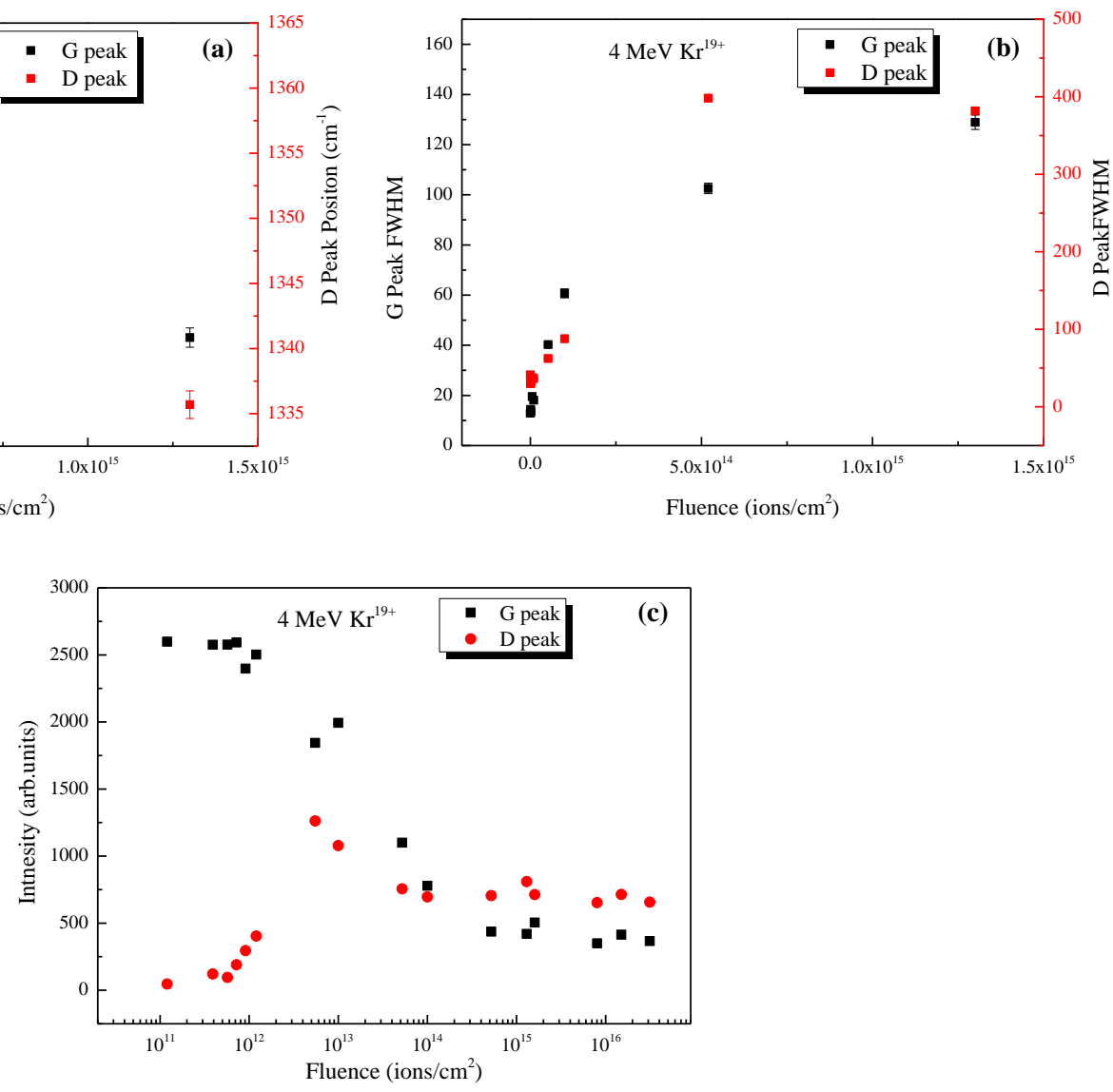
Fig. 2-The evolution of (a) peak center; (b) FWHM and (c) intensity of G and D peaks obtained from HOPG irradiated by $\mathrm{HCI}\left(\mathrm{Kr}^{19+}\right)$ as a function of fluence.

Fig. 2a shows the $G$ and $D$ peaks shifts towards the lower wave numbers with the increasing fluence. The "red shift" of both $G$ and $D$ peaks indicates that the lattice is under tensile strain. The red-shift caused by tensile stress follows $\omega_{\text {vib }}=\omega_{0}\left[1-(a+r+3) / 2 \times \varepsilon_{l b}\right]$ [36] where $\omega_{\text {vib }}$ and $\omega_{0}$ denote the observed peak position and the peak position for the strain-free bulk sample, respectively. $\varepsilon_{l b}$ is the strain of the carbon-carbon bond of length $l_{b}$. The symbols $a$ and $r$ are attractive and repulsive exponents in Mie-Grunsen potentials in solids. For covalent bonding $a+r=3$ [37]. Tensile strain $\left(\varepsilon_{l b}>0\right)$ reduces $\omega_{0}$ to $\omega_{\text {vib }}$ and leads to the red shift. The tensile strain deduced from the red shift of both $\mathrm{G}$ and $\mathrm{D}$ peaks indicates that the lattice structure was damaged by the projectiles. As shown in Fig $2 b$ and 2c, the intensity of $G$ peak decreases with increasing fluence, while the full width at half maximum (FWHM) increases with the fluence. The intensity and FWHM of the D peaks (Fig. 2b and 2c) increase with the ion fluence, which is in agreement with previous works [38-41]. J. Liu et al. [4] analyzed the evolution of G and D peak intensities and discovered a decrease of the crystal domain size at higher ion fluences. This suggest that the growing D peak is an indicator of the accumulation of irradiation-induced defects.

In order to extract quantitative information about the defects evolution in irradiated HOPG the Raman spectra were further analyzed. The intensity ratio of D peak to $G$ peak $\left(I_{\mathrm{D}} / I_{\mathrm{G}}\right)$ is correlated to the structural evolution between the two extremes of graphite and tetrahedral amorphous carbon. The in-plane crystallite size $L_{\alpha}$ can be determined quantitatively by $L_{\alpha}=\mathrm{C} \times\left(I_{\mathrm{D}} / I_{\mathrm{G}}\right)^{-1}$ [42-45], where C is a variable parameter dependent on the laser excitation wavelength. In several literatures, $I_{\mathrm{D}} / I_{\mathrm{G}}$ has been denoted as the area ratio. As pointed out by L.G. Cançado et al. [46], for small disorders or perturbations, the area ratio should be considered since the area under each peak represents the probability of the whole process and the intensity ratio gives much larger errors. However, for large disorder, it is more informative to decouple the information on peak intensity and full width at half maximum. In this paper, we have analyzed the evolution of intensity ratio as a function of fluence to investigate the irradiation effects in HOPG and graphene. 

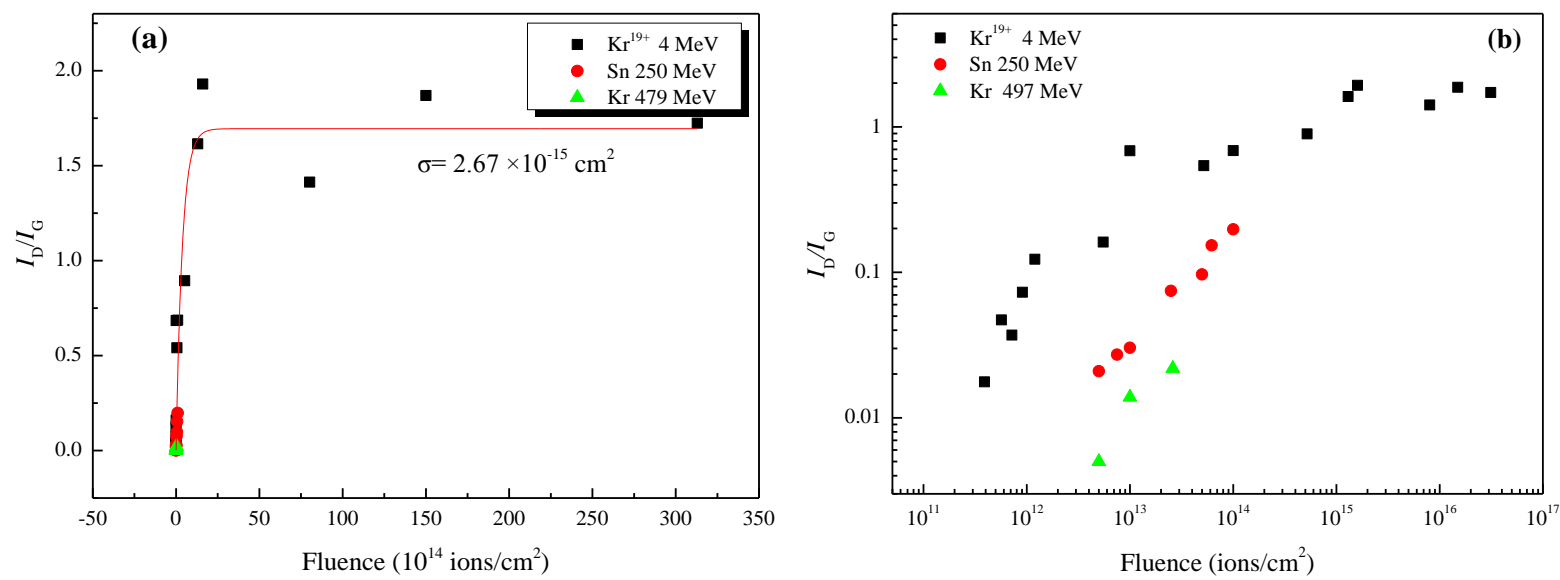

Fig. 3 - (a) The evolution of $I_{\mathrm{D}} / I_{\mathrm{G}}$ from Raman spectra of the HOPG irradiated by HCI $\left(\mathrm{Kr}^{19+}\right)$ and $\mathrm{SHI}(\mathrm{Sn}, \mathrm{Kr})$ as a function of fluence. The data of HOPG irradiated by $\mathrm{Kr}^{19+}$ is fitted according to the relation $f_{(\varphi)}=\alpha[1-\exp (-\sigma \times \varphi)]$ (red line); (b) $I_{\mathrm{D}} / I_{\mathrm{G}}$ versus fluence is plotted on log-log scale for the better comparison.

The intensity ratio $I_{\mathrm{D}} / I_{\mathrm{G}}$ for different ions as a function of fluence is shown in Fig. 3. For a given ion species, the ratio $I_{\mathrm{D}} / I_{\mathrm{G}}$ increases significantly with the increasing fluence. At the same fluence, $I_{\mathrm{D}} / I_{\mathrm{G}}$ is larger for $250 \mathrm{MeV} \mathrm{Sn}$ ions than that of $497 \mathrm{MeV} \mathrm{Kr}$ ions (Fig. 3b). This is related to the different electronic energy losses, $(\mathrm{d} E / \mathrm{d} x)_{\mathrm{e}}$, of the ion beams. As shown in Table.1, the $(\mathrm{d} E / \mathrm{d} x)_{\mathrm{e}}$ is 16.5 and $9.9 \mathrm{keV} / \mathrm{nm}$ for $\mathrm{Sn}$ and $\mathrm{Kr}$, respectively. This agrees well with previous experiments that showed an increase of irradiation damage with increasing $(\mathrm{d} E / \mathrm{d} x)_{\mathrm{e}}[4,47,48]$. Moreover, the velocity effect has been observed in various materials [49-51], and in HOPG in our previous work [39]. The velocity effect was detected in HOPG irradiated by SHI (Bi) with almost the same $(\mathrm{d} E / \mathrm{d} x)_{\mathrm{e}}$, which indicates that besides ion fluence and $(\mathrm{d} E / \mathrm{d} x)_{\mathrm{e}}$, the ion velocity should also be considered in the irradiation effects of swift heavy ions in graphite. The relative velocity $\beta(\beta=v / c)$ is about 0.07 and 0.11 for $\mathrm{Sn}$ and $\mathrm{Kr}$ (shown in Table 1). For the low velocity ions, the energy deposition is more localized, which will lead to more damage in irradiated materials. Then the higher $I_{\mathrm{D}} / I_{\mathrm{G}}$ of $\mathrm{Sn}$ ions irradiation may also be related to the lower velocity. As shown in Fig. $3 b, I_{\mathrm{D}} / I_{\mathrm{G}}$ for low energy $\mathrm{HCI}$ $\left(\mathrm{Kr}^{19+}\right)$ with a kinetic energy of $4 \mathrm{MeV}$ is larger as compared to both types of SHI ( $\mathrm{Sn}$ and $\mathrm{Kr}$ ) irradiations. Two possible factors should be taken in to consideration. First $(\mathrm{d} E / \mathrm{d} x)_{\mathrm{n}}$ of $\mathrm{Kr}^{19+}$ is relatively higher than that of the other two ion types. The second factor is the synergistic effects of $(\mathrm{d} E / \mathrm{d} x)_{\mathrm{e}}$ and Ep of medium energy HCI. As concluded by SRIM (Table. 1), the $(\mathrm{d} E / \mathrm{d} x)_{\mathrm{n}}$ of SHI (Sn and $\mathrm{Kr}$ ) and $\mathrm{HCI}\left(\mathrm{Kr}^{19+}\right)$ are $3.2 \times 10^{-2} \mathrm{keV} / \mathrm{nm}, 0.8 \times 10^{-2} \mathrm{keV} / \mathrm{nm}$ and $36 \times 10^{-2} \mathrm{keV} / \mathrm{nm}$, respectively. This indicates that the damage due to nuclear collisions induced by HCI is larger than that of induced 
by SHI. Damage cross-section is an important parameter for a direct measurement of the damage level of irradiation in the target material. M. Toulemonde et al. [52] pointed out that the damage

3 kinetic is rather complex and can be described by a nucleation and growth process based on Avrami

4 formalism and Poisson law [53-55], with damage fraction $D_{\mathrm{f}}=1-\exp \left(-(\sigma \times \phi t)^{\mathrm{n}}\right)$, where $\phi t$ is the ion

5 fluence, $\sigma$ is the damage cross section and $\mathrm{n}$ is different for various projectile energies. The

6 damage cross section for single ion collision can be roughly deduced from the Raman spectra by using the formula $f_{(\varphi)}=\alpha[1-\exp (-\sigma \times \varphi)]$ as suggested by S. Mathew et al. [56], where $f_{(\varphi)}$ is the

Raman peak intensity ratio $I_{\mathrm{D}} / I_{\mathrm{G}}$ after irradiation of fluence $\varphi, \sigma$ is the damage cross section whereas $\sigma$ and $\alpha$ are the fitting parameters in the equation. As shown in Fig. 3a, the damage cross section $\sigma$ for $4 \mathrm{MeV} \mathrm{Kr}{ }^{19+}$ deduced from Fig. 3a by fitting $I_{\mathrm{D}} / I_{\mathrm{G}}$ data is $(2.67 \pm 1.10) \times 10^{-15} \mathrm{~cm}^{2}$.

The potential energy Ep of $\mathrm{HCI}\left(4 \mathrm{MeV} \mathrm{Kr}^{19+}\right)$ in our case is about $5.6 \mathrm{keV}$ and Ep is normally deposited on HOPG surface with-in initial femtoseconds [57]. As reported by Y. Y. Wang et al. [3], the deposition depth of Ep of medium energy ( 3 and $5 \mathrm{MeV}$ ) $\mathrm{Xe}$ ions in $\mathrm{CaF}_{2}$ is about $4.1 \mathrm{~nm}$. We can deduce that the Ep deposition density of $4 \mathrm{MeV} \mathrm{Kr}^{19+}$ in HOPG must be of the order of several $\mathrm{keV} / \mathrm{nm}$. The $(\mathrm{d} E / \mathrm{d} x)_{\mathrm{e}}$ of SHI $(\mathrm{Sn}$ and $\mathrm{Kr})$ and $\mathrm{HCI}\left(\mathrm{Kr}^{19+}\right)$ are $16.5 \mathrm{keV} / \mathrm{nm}, 9.9 \mathrm{keV} / \mathrm{nm}$ and 2.4 $\mathrm{keV} / \mathrm{nm}$. According to a study reported by Y.Y. Wang et al. [3] for medium energy (3 and $5 \mathrm{MeV}$ ) highly charged ions $\left(\mathrm{Xe}^{22+}\right.$ to $\left.\mathrm{Xe}^{30+}\right)$, both mechanisms of energy deposition (Ep and $\left.(\mathrm{d} E / \mathrm{d} x)_{\mathrm{e}}\right)$ in the target surface seem to contribute in an additive way. Thus we can say that Ep added with the (dE/dx)e of $\mathrm{Kr}^{19+}$ may be comparable or even more than the $(\mathrm{d} E / \mathrm{d} x)_{\mathrm{e}}$ of $\mathrm{SHI}(\mathrm{Sn}$ and $\mathrm{Kr})$ and induce lager $I_{\mathrm{D}} / I_{\mathrm{G}}$

\subsection{Irradiation effects in Graphene}

The monolayer graphene was identified by the Microscopic Raman Spectrometer. As shown in Fig. 4a, the virgin sample has two prominent features: the $G$ peak $\left(\sim 1582 \mathrm{~cm}^{-1}\right)$ and the $2 \mathrm{D}$ peak $\left(\sim 2683 \mathrm{~cm}^{-1}\right)$. According to R. J. Nemanich et al. [58], the G peak is assigned to the Raman-active $\mathrm{E}_{2 \mathrm{~g}}$ mode in the first-order spectrum, and the $2 \mathrm{D}$ peak is assigned to the second order of zone-boundary phonons. It is possible to identify the number of graphene layers by Raman spectroscopy, especially monolayers and bilayers [59]. 

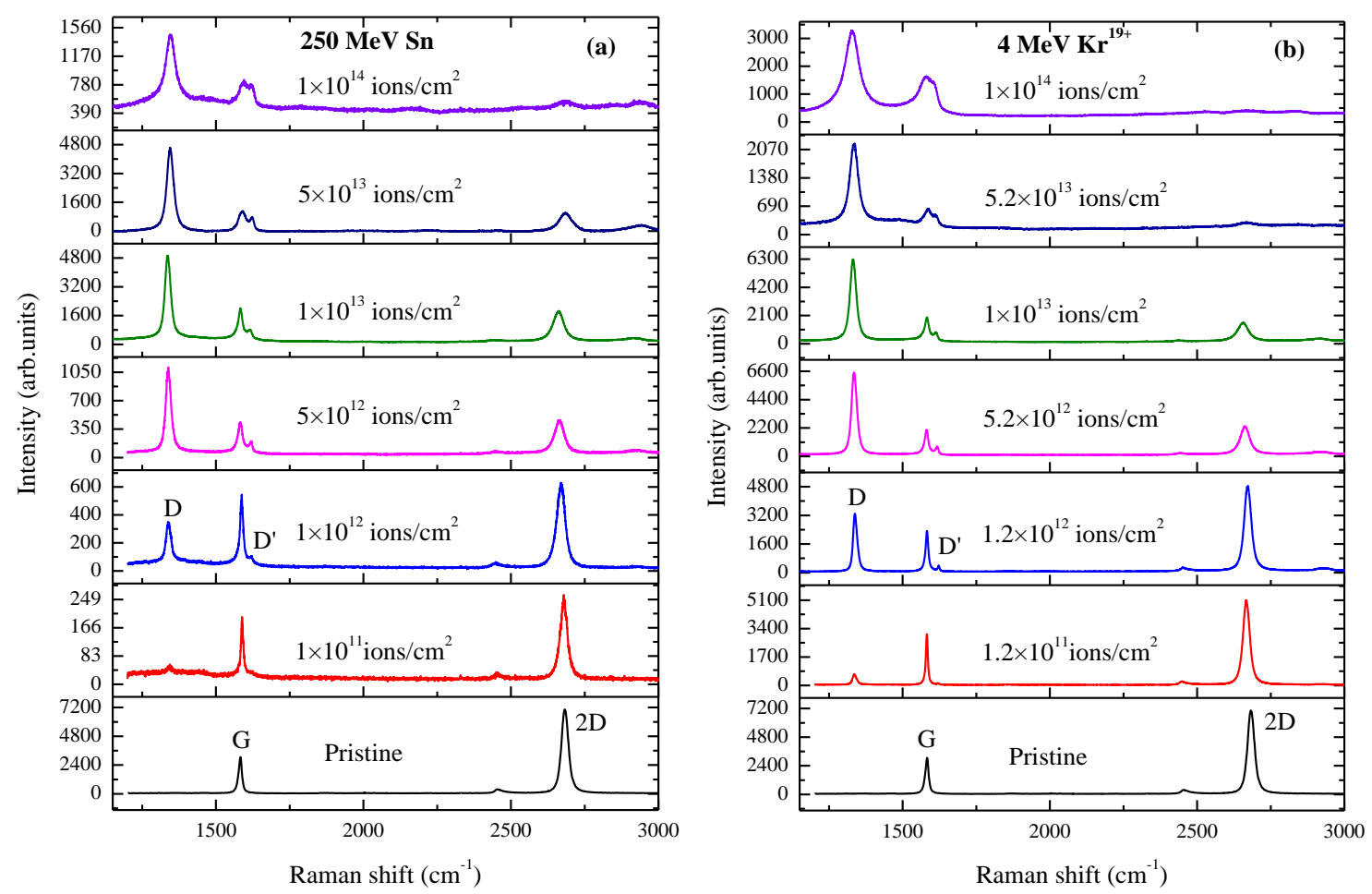

Fig. 4 - Raman spectra of pristine graphene and graphene irradiated by (a) $250 \mathrm{MeV} \mathrm{Sn}$ and (b) $4 \mathrm{MeV} \mathrm{Kr}^{19+}$ with different ion fluences.

According to Fig. 4a, the 2D Raman band of pristine sample is symmetrical, and the intensity of the $2 \mathrm{D}$ peak is twice as much as the intensity of the $\mathrm{G}$ peak, which is an indicator of monolayer graphene. Atomic force microscope (AFM) is a direct method to identify single and multilayer graphene. In our previous work [60], AFM (MFP-3D-SA) was used to confirm the thickness values of graphene sheets and thin graphite films. The thickness of monolayer graphene, confirmed by Raman spectroscopy, is about $0.9 \mathrm{~nm}$, which is in agreement with the result reported by Novoselov et al. [26] The supporting evidence provided by AFM makes sure that the monolayer graphene could be screened out by Raman spectra.

Fig. 4a and 4b show the Raman spectra of graphene irradiated by SHI and HCI, respectively. Both types of ion irradiation lead to the appearance of the D peak $\left(\sim 1338 \mathrm{~cm}^{-1}\right)$ and the $\mathrm{D}^{\prime}$ peak $\left(\sim 1622 \mathrm{~cm}^{-1}\right)$. As mentioned earlier, these two peaks indicate that disorders have been introduced into the graphene lattice by the irradiation. The intensity and FWHM of the D peak and the $\mathrm{D}^{\prime}$ peak increase with ion fluence indicating the accumulation of disorders. In order to obtain quantitative information on the defect accumulation process, all Raman spectra were analyzed using Origin 8.5 Lorentz peak-fitting procedure. The evolution of $I_{\mathrm{D}} / I_{\mathrm{G}}$ as function of fluence is shown in Fig. 5. 

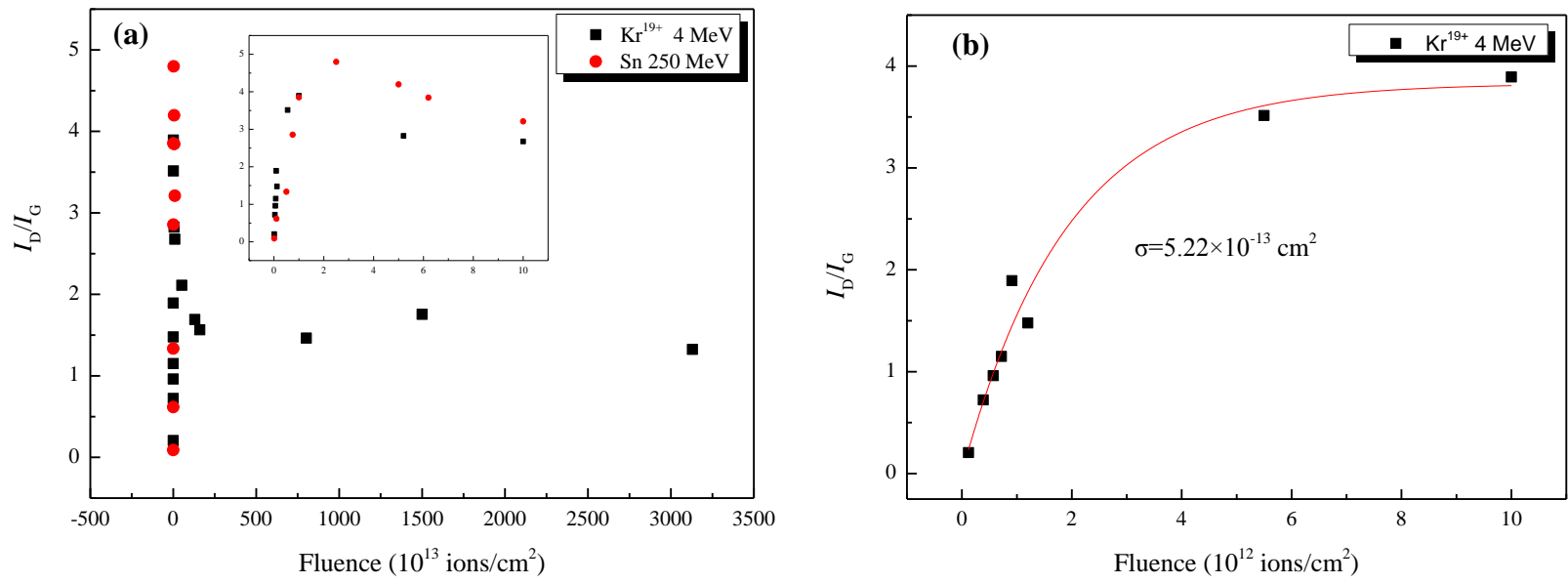

Fig. 5- (a) The evolution of $I_{\mathrm{D}} / I_{\mathrm{G}}$ obtained from graphene irradiated by $\mathrm{HCI}\left(\mathrm{Kr}^{19+}\right)$ and $\mathrm{SHI}(\mathrm{Sn})$ with different fluence;

(b) Variation of $I_{\mathrm{D}} / I_{\mathrm{G}}$ of $\mathrm{HCI}$ irradiated graphene with fitting curve (red line) according to the function

$$
f_{(\varphi)}=\alpha[1-\exp (-\sigma \times \varphi)] .
$$

For graphene irradiated with $250 \mathrm{MeV}$ Sn ions, $I_{\mathrm{D}} / I_{\mathrm{G}}$ increases with fluence up to $2 \times 10^{13}$ ions $/ \mathrm{cm}^{2}$, and then decreases with further irradiation. Almost the same trend was found for $4 \mathrm{MeV}$ $\mathrm{Kr}^{19+}$ ions with a maximum at about $1 \times 10^{13}$ ions $/ \mathrm{cm}^{2}$ (inset of Fig. 5a). Moreover, the $I_{\mathrm{D}} / I_{\mathrm{G}}$ obtained from HCI impacted graphene get saturation when the fluence surpasses $1 \times 10^{15}$ ions $/ \mathrm{cm}^{2}$ (Fig. 5a). The damage cross sections $\sigma$ of $\mathrm{Kr}^{19+}$ in graphene could be deduced from Raman spectra by using the formula $f_{(\varphi)}=\alpha[1-\exp (-\sigma \times \varphi)]$ (discussed in section 3.1). As shown in Fig. 5b, the variation of $I_{\mathrm{D}} / I_{\mathrm{G}}$ as a function of ion fluence could be best fitted with $\sigma=(5.22 \pm 0.67) \times 10^{-13} \mathrm{~cm}^{-2}$. What needs to be mentioned is the procedure was restricted to data of fluences lower than $1 \times 10^{13}$ ions $/ \mathrm{cm}^{2}$, where the damage originates from the impact of individual ions [52]. The maximum $I_{\mathrm{D}} / I_{\mathrm{G}}$ of graphene irradiated by SHI and HCI is about 4.8 and 3.9, respectively. It is interesting to note that, at low fluence, $I_{\mathrm{D}} / I_{\mathrm{G}}$ of graphene irradiated by HCI is larger than that of graphene irradiated by SHI. When the fluence surpasses $1 \times 10^{13}$ ions $/ \mathrm{cm}^{2}$, at which the $I_{\mathrm{D}} / I_{\mathrm{G}}$ for HCI gets the maximum value, larger $I_{\mathrm{D}} / I_{\mathrm{G}}$ for SHI shows up. This is comparatively different when compared with the differences of $I_{\mathrm{D}} / I_{\mathrm{G}}$ observed in HOPG for the different kinds of ion irradiations. This must be related to the unique monolayered structure of graphene. The theoretical thickness of monolayer graphene is $0.34 \mathrm{~nm}$, and the present theory for bulk materials does not necessarily valid in graphene. Further work is required to figure out the energy deposition mechanisms in these materials.

According to the simulations of graphene carried out by O. Lehtinen et al. [61], the most easily 
introduced defects in the graphene lattice are point defects. Simulations performed by A. V.

Krasheninnikov et al. [62] proved that the most common defects induced in carbon nanotubes, which could be viewed as rolled graphene, are the vacancies. M. Ouyang et al. [63] observed vacancies in single-walled nanotube samples by STM. Latter, A. Hashimoto et al. [64] used high-resolution transmission electron microscopy (HR-TEM) to study defects in graphene and confirmed defects such as topological defects; vacancies and adatoms are stable in graphene. J.C. Meyer et al. [65] presented quantitative analysis of electron-beam-induced displacements of carbon atoms in single-layer graphene. A phenomenological model proposed by Lucchese et al. [21] suggested that the evolution of $I_{\mathrm{D}} / I_{\mathrm{G}}$ depends on the density of vacancy defects. In order to explore further about the defects accumulation processes in graphene, the average distance between two adjacent defects, $L_{\mathrm{d}}$, is introduced into this work. $L_{\mathrm{d}}$ is defined as $L_{\mathrm{d}}=1 / \sqrt{\varphi}$, where $\varphi$ is the ion beam fluence [21, 23].

The evolution of $I_{\mathrm{D}} / I_{\mathrm{G}}$ collected from the graphene irradiated by different kind of energetic ions are almost the same: $I_{\mathrm{D}} / I_{\mathrm{G}}$ increases with decreasing $L_{\mathrm{d}}$ until $L_{\mathrm{d}}$ surpasses a critical value, and then $I_{\mathrm{D}} / I_{\mathrm{G}}$ decreases with further decreasing of $L_{\mathrm{d}}$. Similar results have been reported by M.M. Lucchese et al. [21], where the evolution process of $I_{\mathrm{D}} / I_{\mathrm{G}}$ with $L_{\mathrm{d}}$ was proposed as:

$I_{\mathrm{D}} / I_{\mathrm{G}}=C_{\mathrm{A}} \frac{r_{\mathrm{A}}^{2}-r_{\mathrm{S}}^{2}}{r_{\mathrm{A}}^{2}-2 r_{\mathrm{S}}^{2}}\left[\exp \left(-\pi r_{\mathrm{S}}^{2} / L_{\mathrm{d}}^{2}\right)-\exp \left(-\pi\left(r_{\mathrm{A}}^{2}-r_{\mathrm{S}}^{2}\right) / L_{\mathrm{d}}^{2}\right)\right]+C_{\mathrm{S}}\left[1-\exp \left(-\pi r_{\mathrm{S}}^{2} / L_{\mathrm{d}}^{2}\right)\right]$

Where $r_{\mathrm{A}}$ and $r_{\mathrm{S}}$ are the radii of two different regions defined as "structurally disordered" S-region and "activated" A-region, respectively. Within the radius $r_{\mathrm{S}}$, structural disorder occurs due to the bombarding of energetic ions. For the distance larger than $r_{\mathrm{S}}$ but smaller than $r_{\mathrm{A}}$, the lattice structure is preserved as a result of the close proximity to disordered regions. The A-regions shall contribute more strongly to the D band, while the S-regions will contribution little to the $\mathrm{D}$ band due to the breakdown of lattice structure itself. The relaxation length for resonant Raman scattering in graphene is $\ell=r_{\mathrm{A}}-r_{\mathrm{S}} \mathrm{nm}$. $C_{\mathrm{A}}$ is a measure of the maximum possible value of the $I_{\mathrm{D}} / I_{\mathrm{G}}$ ratio in graphene. $C_{\mathrm{A}}$ could be defined by the electron-phonon matrix elements between the $\Gamma$ and $K$ points $[21,66] . C_{\mathrm{S}}$ is the minimum value of $I_{\mathrm{D}} / I_{\mathrm{G}}$ in highly disordered limit, which has not been addressed theoretically yet.

As reported by J. Liu et al. [5], a critical $(\mathrm{d} E / \mathrm{d} x)_{\mathrm{e}}$ threshold of $7.3 \pm 1.5 \mathrm{keV} / \mathrm{nm}$ for the latent track formation in HOPG was detected. For the $(\mathrm{d} E / \mathrm{d} x)_{\mathrm{e}}$ values of 9 to $18 \mathrm{keV} / \mathrm{nm}$, there exists a 
1 large discrepancy between the number density of detected tracks and ion fluence. A probability of one is only found for an energy loss above $\sim 18 \mathrm{keV} / \mathrm{nm}$. J.C. Meyer et al. [65] made accurate measurements of atomic displacement cross sections for carbon atoms in single-layer graphene. They pointed out that under $80 \mathrm{keV}$ irradiation, no vacancies could be formed in pristine areas up to very

5 high doses (beyond $10^{10} e^{-} / \mathrm{nm}^{2}$ ). The cross section smoothly increases from practically zero $\left(10^{-4}\right.$ barn) at $80 \mathrm{keV}$ to 0.2 barn at $100 \mathrm{keV}$. Then considering the possibility that each ion is not inducing defect in graphene, we infer that a defect creation probability $P$ is required to calculate the average distance between defects. Thus, the modified distance $L_{\mathrm{d}}{ }^{\prime}$ can be expressed as:

$$
L_{\mathrm{d}}{ }^{\prime}=1 / \sqrt{P \times \varphi}=L_{\mathrm{d}} / \sqrt{P}
$$

Furthermore, the corrected expression for $I_{\mathrm{D}} / I_{\mathrm{G}}$ can be modified as:

$I_{\mathrm{D}} / I_{\mathrm{G}}=C_{\mathrm{A}} \frac{r_{\mathrm{A}}^{2}-r_{\mathrm{S}}^{2}}{r_{\mathrm{A}}{ }^{2}-2 r_{\mathrm{S}}^{2}}\left[\exp \left(-\pi r_{\mathrm{S}}^{2} P / L_{\mathrm{d}}^{2}\right)-\exp \left(-\pi\left(r_{\mathrm{A}}^{2}-r_{\mathrm{S}}^{2}\right) P / L_{\mathrm{d}}{ }^{2}\right)\right]+C \mathrm{~s}\left[1-\exp \left(-\pi r_{\mathrm{S}}^{2} P / L_{\mathrm{d}}^{2}\right)\right]$

Fig. 6 (a) shows the evolution of $I_{\mathrm{D}} / I_{\mathrm{G}}$ obtained from irradiated monolayer graphene samples as a function of $L_{\mathrm{d}}$, with a $\log -\log$ scale plot as shown in Fig. 6 (b). Eq. (3) was fitted to the graphene $I_{\mathrm{D}} / I_{\mathrm{G}}$ data for different kind of ion irradiation experiments. Using the parameters $C_{\mathrm{A}}=5.66, C_{\mathrm{S}}=2.62$, $r_{\mathrm{A}}=4.68, r_{\mathrm{S}}=1.79$ and $P=0.20$, Eq. (3) describes the experimental data of SHI (Sn) irradiated graphene (red circular dots) very well. As shown in Fig. 6a, the maximum value of $I_{\mathrm{D}} / I_{\mathrm{G}}$ is about 5 and $C_{\mathrm{A}}=5.66$ is in accordance with this value. In this work, the minimum value of $I_{\mathrm{D}} / I_{\mathrm{G}}$ in highly disordered limit can't be detected as the fluence is not high enough. It can be inferred from the value $C_{\mathrm{S}}=2.62$ in phenomenological model that the minimum values of $I_{\mathrm{D}} / I_{\mathrm{G}}$ in graphene is about 2.62 . The Raman relaxation length $\ell=r_{\mathrm{A}}-r_{\mathrm{S}}=2.89 \mathrm{~nm}$. It is interesting to note that $\ell=2 \mathrm{~nm}$ induced by low energy heavy ions $\left(\mathrm{Ar}^{+}\right)$[7] is comparable with $\ell=2.68 \mathrm{~nm}$ induced by SHI (Sn). One possible reason for the same $\ell$ should be the unique single-layer atom structure of graphene. The damage cross section does not vary much for various ion velocities and the velocity effects in graphene can be neglected. This is in good agreement with our previous work [39], in which the velocity effect was found in HOPG but never detected in graphene. 

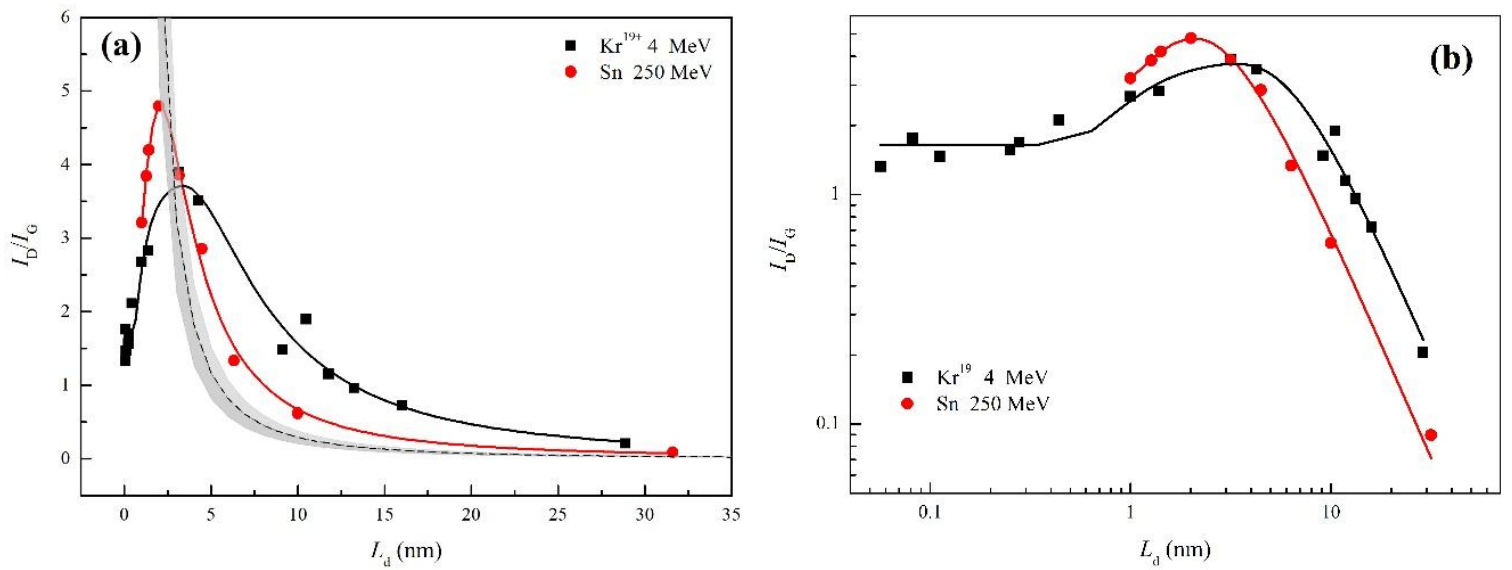

Fig. 6 - (a) The evolution of $I_{\mathrm{D}} / I_{\mathrm{G}}$ obtained from graphene irradiated by $\mathrm{HCI}\left(\mathrm{Kr}^{19+}\right)$ and SHI (Sn) with different fluence. The dashed line is the relationship reported by Cançado et al. [46] (b) a log-log scale plot of $I_{\mathrm{D}} / I_{\mathrm{G}} \mathrm{vs}$. $L_{\mathrm{d}}$

The defect creation probability $P=0.20$ is confirmed by fitting the experimental data, indicating that not every Sn ion introduces a defect in graphene. According to Cançado et al. [45] the crystallite size $L_{\alpha}$ of the nanographite samples could be given by $L_{\alpha}(\mathrm{nm})=560 \times E_{l}^{-4} \times\left(I_{\mathrm{D}} / I_{\mathrm{G}}\right)^{-1}$, where $E_{l}$ is the excitation laser energy used in Raman experiment in $\mathrm{eV}$ units. Later Cançado et al. expanded this work [46] and reported that in the low-defect density regime ( $\left.L_{\alpha} \geq 10 \mathrm{~nm}\right)$, in which the D band scattering is proportional to the number of point defects, $L_{\alpha}$ of the graphene follows an empirical relation of the form $L_{\alpha}^{2}\left(\mathrm{~nm}^{2}\right)=(4.3 \pm 1.3) \times 10^{3} \times E_{l}^{-4} \times\left(I_{\mathrm{D}} / I_{\mathrm{G}}\right)^{-1}$. In this work, the excitation wavelength used was $532 \mathrm{~nm}$, thus the value of $E_{l}$ comes out to be $2.33 \mathrm{eV}$ and $L_{\alpha}=L_{\mathrm{d}}{ }^{\prime}=L_{\mathrm{d}} / \sqrt{P}$, for $250 \mathrm{MeV}{ }^{112} \mathrm{Sn}$ ions impacted graphene $L_{\alpha}=L_{\mathrm{d}} / \sqrt{0.2}$. The empirical relation is shown in Fig. 6a (dashed line) where the shadow area accounts for the error range. The empirical relation is proved to be efficient in graphene irradiated by $250 \mathrm{MeV}{ }^{112} \mathrm{Sn}$ ions in the regime $L_{\alpha} \geq 10 \mathrm{~nm}$. This confirmed that the defect creation probability $P=0.2$ is reasonable, however, more experimental work needs to be done to confirm P.

The fitting curve of experimental data detected from $\mathrm{HCI}\left(\mathrm{Kr}^{19+}\right)$ irradiated graphene (black square dots) was given by Eq. (3) with parameters $C_{\mathrm{A}}=3.92, C_{\mathrm{S}}=2.15, r_{\mathrm{A}}=6.94, r_{\mathrm{S}}=1.22$ and $P=0.34$. As shown in Fig. 6a, for $\mathrm{Kr}^{19+}$ irradiated graphene, the maximum value of $I_{\mathrm{D}} / I_{\mathrm{G}}$ is about 4 , and the minimum value of $I_{\mathrm{D}} / I_{\mathrm{G}}$ in highly disordered limit is about $1.75 . C_{\mathrm{A}}=3.92, C_{\mathrm{S}}=2.15$ are approximated to the maximum and minimum values of $I_{\mathrm{D}} / I_{\mathrm{G}}$. The Raman relaxation length $(\ell=5.72 \mathrm{~nm})$ is larger 
1 than $\ell=2 \mathrm{~nm}$ induced by the low energy heavy ions $\left(\mathrm{Ar}^{+}\right)$as well as $\ell=2.89 \mathrm{~nm}$ induced by SHI (Sn).

2 The bigger $\ell$ detected in graphene irradiated by HCI infers a larger area of the activated region. This must be ascribed to the large potential energy Ep of the HCI. When a HCI approaches the graphene sheet, the HCI accumulates large number of electrons into its high Rydberg orbitals and subsequently emits the electrons by Auger decay processes $[67,68]$. This process repeats many times until the HCI become nearly neutralized. The transfer of potential energy of HCI initially excites the electronic structure of the graphene that may lead to the larger area of activated region around the latent track.

\subsection{Comparison of irradiation effects in HOPG and graphene}

As discussed in section 3.2, the irradiation effects in HOPG and graphene are different from each other. At the same ion fluence, larger $I_{\mathrm{D}} / I_{\mathrm{G}}$ was found in the case of HCI than that of SHI in HOPG, while such trend was not detected in the case of graphene. Difference irradiation effects in HOPG and graphene caused by SHI have been discussed in our earlier work [39]. Due to the special 14 single atomic layer structure of graphene, the irradiation fluence threshold for the D band of graphene is significantly lower than that of HOPG. The velocity effect was found in HOPG but this effect didn't detect in graphene. Under the same irradiation conditions, larger defect density was found in graphene indicating that monolayer graphene is easier to damage than the bulk HOPG by by HCI.

The $I_{\mathrm{D}} / I_{\mathrm{G}}$ of graphene and HOPG are plotted as a function of ion fluence as shown in Fig. 7. It is seen that $I_{\mathrm{D}} / I_{\mathrm{G}}$ for HOPG increases with ion fluence and reaches a plateau after $10^{15} \mathrm{ions} / \mathrm{cm}^{2}$. While in the case of graphene, $I_{\mathrm{D}} / I_{\mathrm{G}}$ increases with the fluence at the beginning, and then begins to decrease for the fluence larger than $1 \times 10^{13}$ ions $/ \mathrm{cm}^{2}$, and finally achieves saturation after the ion fluence of $10^{15}$ ions $/ \mathrm{cm}^{2}$. The saturation values of $I_{\mathrm{D}} / I_{\mathrm{G}}$ in graphene and $\mathrm{HOPG}$ are almost the same $\left(I_{\mathrm{D}} / I_{\mathrm{G}} \approx 1.8\right)$. 

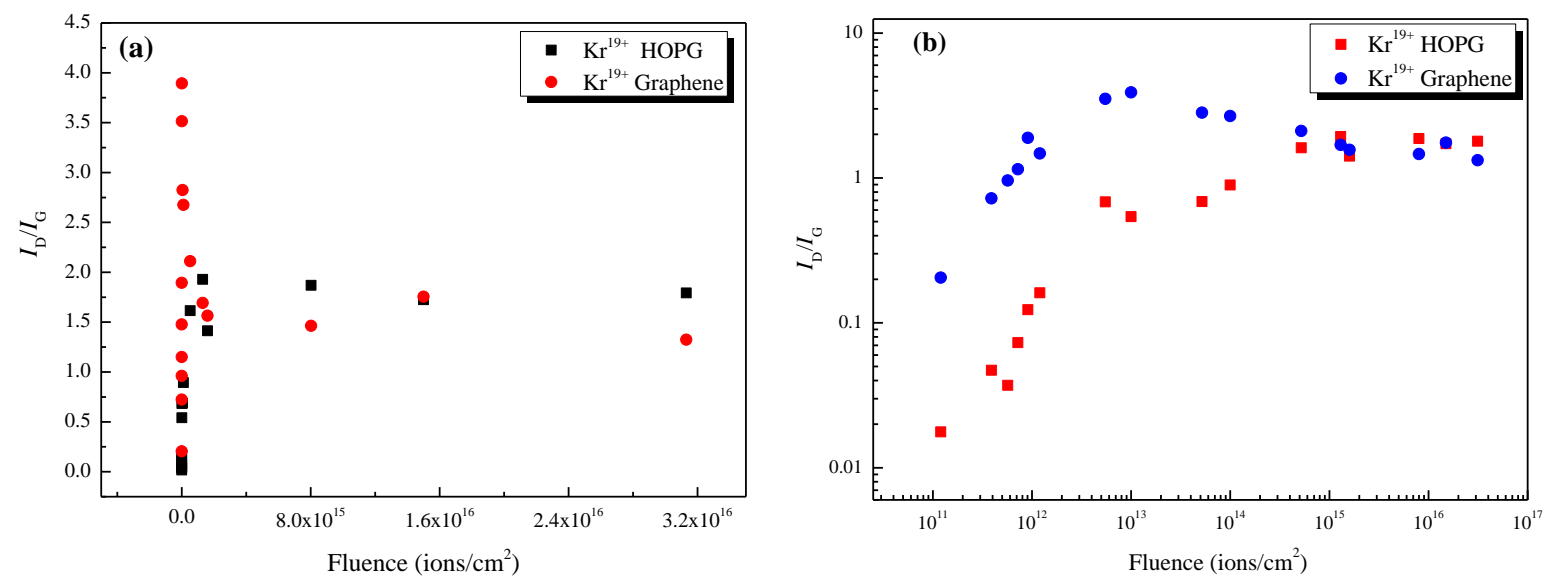

Fig. 7 - (a) The evolution of $I_{\mathrm{D}} / I_{\mathrm{G}}$ for monolayer graphene and HOPG samples irradiated by $\mathrm{Kr}^{19+}$ with different fluences. (b) The evolution of $I_{\mathrm{D}} / I_{\mathrm{G}}$ in $\log -\log$ plot for better comparison.

The plot of $I_{\mathrm{D}} / I_{\mathrm{G}}$ vs. $L_{\mathrm{d}}$ is shown in Fig. 8. As shown in Fig. 8a, the $I_{\mathrm{D}} / I_{\mathrm{G}}$ ratio of irradiated graphene ranges from 0.1 to 4.0 , which is much larger than that of the HOPG case $(0.02-2.0)$. This predicts that the response of graphene and HOPG are different for HCI irradiation. In section 3.1 and 3.2 we discussed about the damage cross section $\sigma$ for $4 \mathrm{MeV} \mathrm{Kr}^{19+}$ in $\mathrm{HOPG}$ and graphene. The cross section $\sigma$ in graphene is $(5.22 \pm 0.67) \times 10^{-13} \mathrm{~cm}^{-2}$, which is larger than that of HOPG which is $(2.67 \pm 1.10) \times 10^{-15} \mathrm{~cm}^{2}$. The lager $\sigma$ value in this case indicates that monolayer graphene is much easier to be damaged by HCI irradiation than the bulk graphite, which is in agreement with the results obtained from the SHI irradiation experiments [39].

As shown in Fig. 8a, a turning point (maximum of $I_{\mathrm{D}} / I_{\mathrm{G}} \sim 4$ ) was detected in the evolution process of $I_{\mathrm{D}} / I_{\mathrm{G}}$ with fluence obtained from graphene, while such point was never found in HOPG in the entire fluence range used in this work. The evolution of $I_{\mathrm{D}} / I_{\mathrm{G}}$ is much clear on the log-log scale as shown in Fig. 8b. For graphene, three various regions of the $I_{\mathrm{D}} / I_{\mathrm{G}}$ are evident: (1) the $I_{\mathrm{D}} / I_{\mathrm{G}}$ increases with the decreasing $L_{\mathrm{d}}$ in the region where $L_{\mathrm{d}}>3.2 \mathrm{~nm}$, (2) the decreasing trend in the region where $0.3 \mathrm{~nm}<L_{\mathrm{d}}<3.2 \mathrm{~nm}$, and (3) the stabilized $I_{\mathrm{D}} / I_{\mathrm{G}}$ range for $L_{\mathrm{d}}<0.3 \mathrm{~nm}$. While for HOPG only two regions were detected during the $I_{\mathrm{D}} / I_{\mathrm{G}}$ evolution process: the $I_{\mathrm{D}} / I_{\mathrm{G}}$ increasing region with decreasing $L_{\mathrm{d}}$ for $L_{\mathrm{d}}>0.3 \mathrm{~nm}$, and the stabilized region when $L_{\mathrm{d}}<0.3 \mathrm{~nm}$. The stabilization mentioned in [29, 69] has been attributed to the creation of vacancy clusters due to the overlap of the irradiation induced cascades. 

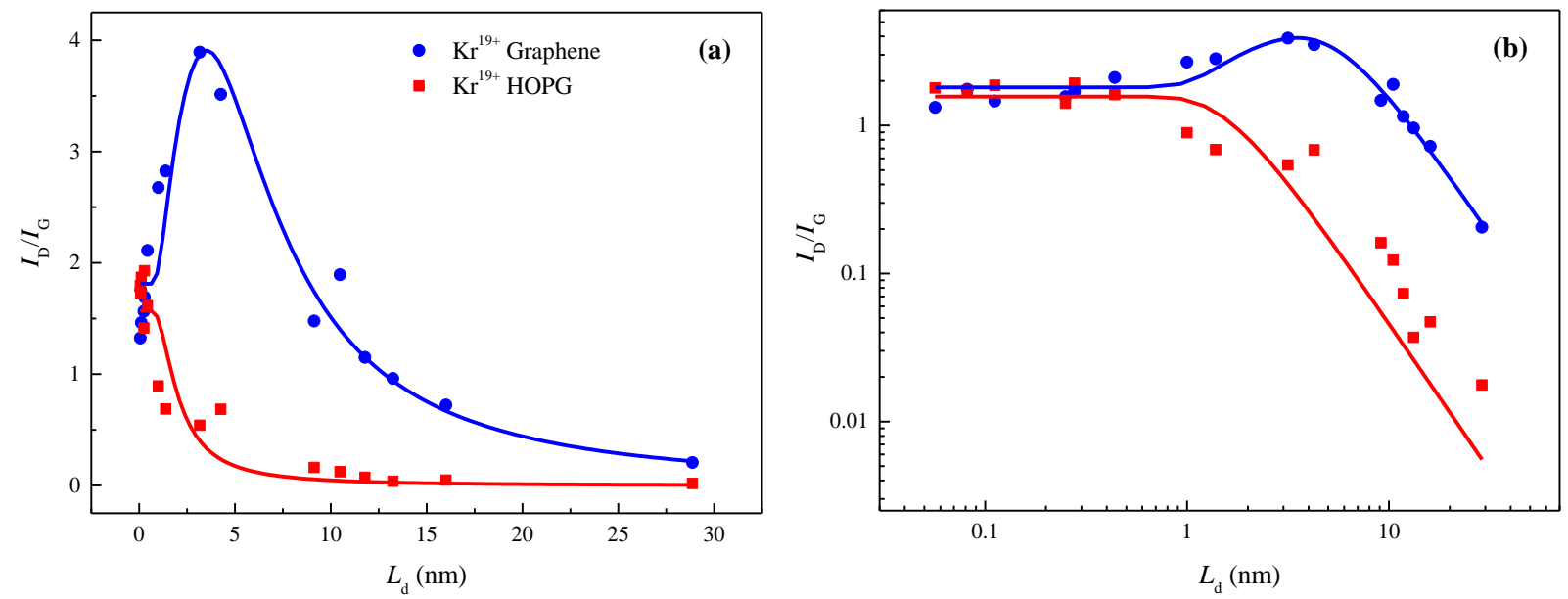

Fig. 8 - (a) Comparison of the $I_{\mathrm{D}} / I_{\mathrm{G}}$ values obtained from $\mathrm{Kr}^{19+}$ irradiated HOPG (square data points) and graphene (circle data points). The solid line of graphene is given by equation (3). The solid line of HOPG plotted according to equation (4) with parameter $C_{\mathrm{A}}=1.59 \pm 0.1 r_{\mathrm{A}}=1.22$ and $P=0.63$. (b) The evolution of $I_{\mathrm{D}} / I_{\mathrm{G}}$ in log-log plot.

As already discussed in part 3.2, Eq. (3) can give perfect fitting of $I_{\mathrm{D}} / I_{\mathrm{G}}$ of graphene. Here we used Eq. (3) to fit values of the $I_{\mathrm{D}} / I_{\mathrm{G}}$ for HOPG. Eq. (3) seems to provide an appropriate fitting of the curve at $r_{\mathrm{S}}=0$ in Fig. $8 \mathrm{a}$ and $8 \mathrm{~b}$.

At $r_{\mathrm{S}}=0$, the evolution equation (Eq. (3)) becomes of the form

$$
I_{\mathrm{D}} / I_{\mathrm{G}}=C_{\mathrm{A}}\left[1-\exp \left(-\pi r_{\mathrm{A}}{ }^{2} P / L_{\mathrm{d}}{ }^{2}\right)\right]
$$

As shown in Fig. 8a, the $I_{\mathrm{D}} / I_{\mathrm{G}}$ ratio of HOPG is fitted by a solid line, which is given by Eq. (4) with parameters $C_{\mathrm{A}}=1.59, r_{\mathrm{A}}=1.22$, and $P=0.63$. According to the phenomenological model [21], $C_{\mathrm{A}}$ is a measure of the maximum possible value of $I_{\mathrm{D}} / I_{\mathrm{G}}$ ratio in graphene. Then in equation $4, C_{\mathrm{A}}$ is related to the maximum value of $I_{\mathrm{D}} / I_{\mathrm{G}}$ in HOPG. The fitting parameter $C_{\mathrm{A}}=1.59$ is consistent with the maximum $I_{\mathrm{D}} / I_{\mathrm{G}}$ shown in Fig. 8a. The fitting parameter $r_{\mathrm{S}}=0$ indicates that the S-region is absent. To understand this, we need to compare the defects in HOPG and graphene using the definitions of S-region and A-region in the phenomenological model.

Fig. 9a shows the nanopore in graphene with diameter of $8 \mathrm{~nm}$ detected in our previous work [70]. Comparing with the definition of S- and A-region in the phenomenological model (referring to Fig. 5a in Ref. [21]), the nanopore in graphene could be inferred to the S-region, in which structural disorder from the impact occurs. The lattice structure around S-region is preserved that corresponds to the A-region. The preserved lattice in A-region will contribute more strongly to the Raman D peak. Due to the breakdown of lattice structure, the S-regions will make little contribution to the D peak. At the very beginning of irradiation, the pristine graphene lattice turns into S-regions and A-regions, 
and the area of A-region increases, which results in the increasing D peak intensity. When the ion fluence surpasses certain value, the adjacent S-regions start to overlap. As the number of impacts

3 increase, the area of S-region increases while that of A-region decreases, which lead to decrease of D peak intensity. A maximum of $I_{\mathrm{D}} / I_{\mathrm{G}}$ arises as a result of the transform process. The position of maximum depends on the fluence, at which the S-region start to dominate the competition process.
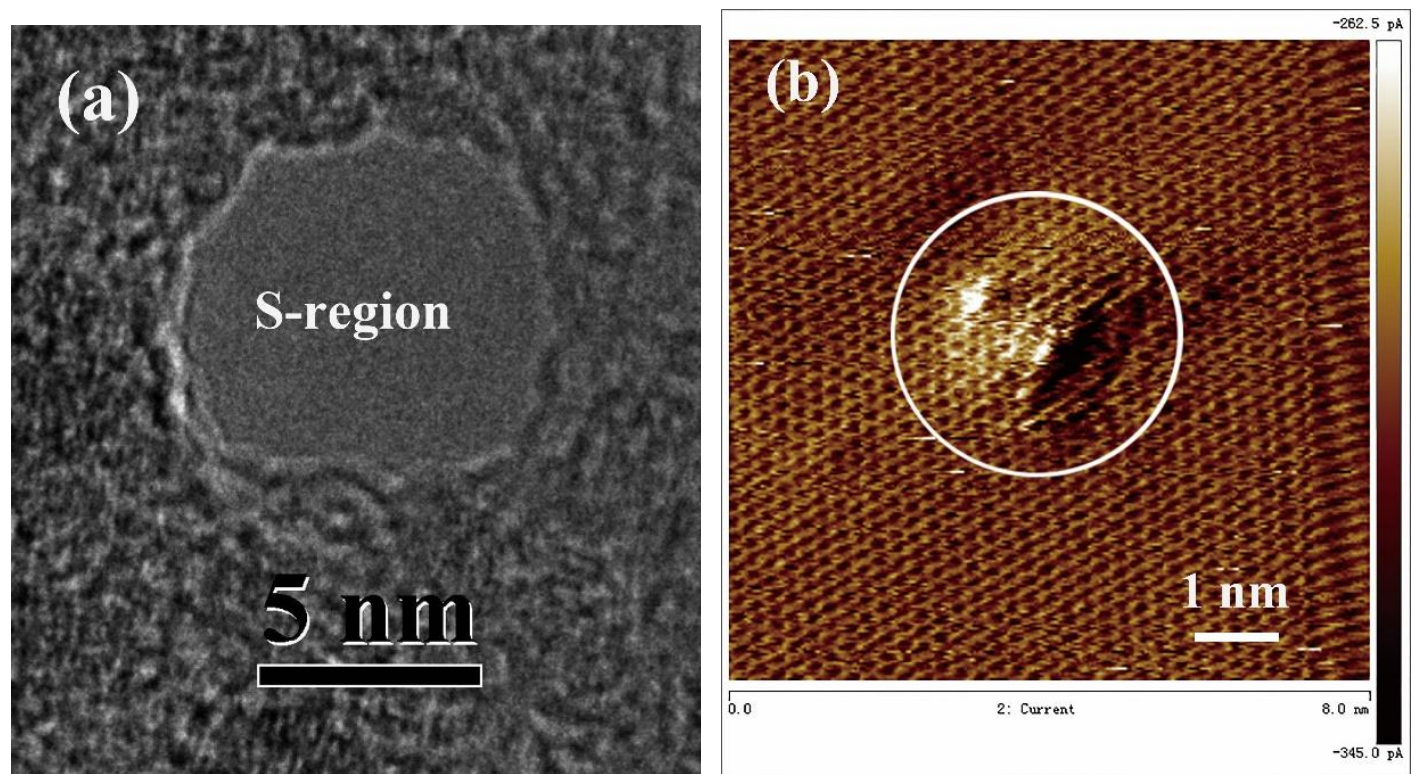

Fig. 9-(a) TEM image of nanopore in graphene quoted from previous work [70]. (b) STM images of HOPG irradiated by $250 \mathrm{MeV}$ Sn with scan area of $8 \mathrm{~nm} \times 8 \mathrm{~nm}$.

In Fig. 9b, the latent track is detected on $250 \mathrm{MeV} \mathrm{Sn} \mathrm{irradiated} \mathrm{graphite} \mathrm{surface,} \mathrm{which} \mathrm{is} \mathrm{in}$ good agreement with previous results obtained by Liu et al. $[4,5,48]$ The atomic scale was reached with a scan area of $8 \mathrm{~nm} \times 8 \mathrm{~nm}$. The defect is nearly in a circular shape. The lattice structure in the circular region has been twisted by the projectile. The twisted lattice structure leads to an enhancement of the D peak. All around the hillock, the graphite lattice is still visible with classical interatomic distance. Comparing with definition of S-region and A-region, the region inside the circle should be A-region, which can enhance the D peak. The S-region with totally destroyed lattice structure must be in the core of the latent track. According to the fitting results the radii of S-region is $r_{\mathrm{S}}=0$, which indicates that almost the whole hillock area produced by HCI on HOPG surface is the activated region and the S-region in HOPG is too small therefore can be ignored. From this it can be concluded that: in graphene, the competing mechanism of "structure disordered" S-region and the "activated" A-region results in the three variation regions of the $I_{\mathrm{D}} / I_{\mathrm{G}}$ of graphene, while in HOPG, no such competing mechanism existed, and no turning point could be found in the evolution process 


\section{Conclusion}

In conclusion, the single layer graphene on $\mathrm{Si} / \mathrm{SiO}_{2}$ substrate and bulk graphite (HOPG) irradiated by SHI and HCI were investigated using Raman Spectrometer. Different irradiation results obtained from the HOPG and graphene samples caused by SHI and HCI irradiation were discussed. Under the same irradiation ion fluences, the greater $I_{\mathrm{D}} / I_{\mathrm{G}}$ ratios were found in HOPG impacted by HCI than that by SHI. This indicates that lager $(\mathrm{d} E / \mathrm{d} x)_{\mathrm{n}}$ of HCI and the synergistic effects of Ep and

$9(\mathrm{~d} E / \mathrm{d} x)_{\mathrm{e}}$ of medium energy HCI have to be considered during the energy deposition process. The

10 Lucchese's phenomenological model was modified to give full line fitting of the experimental data of HOPG and graphene. Comparing the graphene samples irradiated by SHI and HCI, the larger radius of the activated region $r_{\mathrm{A}}$ in $\mathrm{HCI}$ irradiated graphene was observed. By comparing the irradiation effects of HOPG and graphene caused by HCI, it is found that monolayer graphene is much easier to be damaged than the bulk graphite. A turning point was detected in the evolution process of $I_{\mathrm{D}} / I_{\mathrm{G}}$ with the fluence obtained from graphene, while such point was not observed in HOPG in the entire fluence range used in this experiment. According to the improved phenomenological model, the energetic ions may cause both structurally disordered and activated regions in graphene. The competing mechanism of these two regions results in three different regions of $I_{\mathrm{D}} / I_{\mathrm{G}}$ for graphene. In HOPG, however, activated region is induced by energetic ions, only two regions of $I_{\mathrm{D}} / I_{\mathrm{G}}$ variation were detected, which explains the lack of a turning point in the $I_{\mathrm{D}} / I_{\mathrm{G}}$ behavior.

\section{Acknowledgments}

The irradiation work was performed at HIRFL and the $320 \mathrm{kV}$ platform for multi-discipline research with highly charged ions at the Institute of Modern Physics, CAS. We are thankful to the staff of HIRFL and $320 \mathrm{kV}$ platform. We are also thankful to K. Maaz for carefully going through the manuscript. This work was supported by National Science Foundation of China (Nos.11179003, 10975164, 10805062, 11005134 and 11275237). 
[1] Kanjilal D. Swift heavy ion-induced modification and track formation in materials. Curr Sci India. 2001;80(12):1560-6.

[2] Aumayr F, Facsko S, El-Said AS, Trautmann C, Schleberger M. Single ion induced surface nanostructures: a comparison between slow highly charged and swift heavy ions. Journal of physics Condensed matter : an Institute of Physics journal. 2011;23(39):393001.

[3] Wang YY, Grygiel C, Dufour C, Sun JR, Wang ZG, Zhao YT, et al. Energy deposition by heavy ions: additivity of kinetic and potential energy contributions in hillock formation on CaF2. Scientific reports. 2014;4:5742.

[4] Liu J, Hou MD, Trautmann C, Neumann R, Müller C, Wang ZG, et al. STM and Raman spectroscopic study of graphite irradiated by heavy ions. Nucl Instrum Methods Phys Res, Sect B. 2003;212:303-7.

[5] Liu J, Neumann R, Trautmann C, Müller C. Tracks of swift heavy ions in graphite studied by scanning tunneling microscopy. Phys Rev B. 2001;64(18):184115.

[6] Szenes G, Fink D, Klaumünzer S, Pászti F, Péter Á. Ion-induced tracks in Bi4Ge3O12 and Bi12GeO20 crystals. Nucl Instrum Methods Phys Res, Sect B. 2006;245(1):243-5.

[7] Dallanora A, Marcondes TL, Bermudez GG, Fichtner PFP, Trautmann C, Toulemonde M, et al. Nanoporous $\mathrm{SiO}_{2} / \mathrm{Si}$ thin layers produced by ion track etching: Dependence on the ion energy and criterion for etchability. J Appl Phys. 2008;104(2):024307.

[8] Khalfaoui N, Rotaru CC, Bouffard S, Toulemonde M, Stoquert JP, Haas F, et al. Characterization of swift heavy ion tracks in $\mathrm{CaF} 2$ by scanning force and transmission electron microscopy. Nucl Instrum Methods Phys Res, Sect B. 2005;240(4):819-28.

[9] Terada M, Nakamura N, Nakai Y, Kanai Y, Ohtani S, Komaki K-i, et al. Observation of an HCI-induced nano-dot on an HOPG surface with STM and AFM. Nucl Instrum Methods Phys Res, Sect B. 2005;235(1-4):452-5.

[10] Hayderer G, Cernusca S, Schmid M, Varga P, Winter H, Auma F. STM studies of HCI-induced surface damage on highly oriented pyrolytic graphite. Phys Scripta. 2001;92:156-7.

[11] Meguro T, Yamaguchi Y, Fukagawa H, Takai H, Hanano N, Yamamoto Y, et al. Nanoscale modification of electronic states of HOPG by the single impact of HCI. Nucl Instrum Methods Phys Res, Sect B. 2005;235(1-4):431-7.

[12] Sideras-Haddad E, Schenkel T, Shrivastava S, Makgato T, Batra A, Weis CD, et al. Possible diamond-like nanoscale structures induced by slow highly-charged ions on graphite (HOPG). Nucl Instrum Methods Phys Res, Sect B. 2009;267(16):2774-7.

[13] Havancsák K, Birö LP, Gyulai J, Didyk AJ. STM and AFM observations of damage produced by swift Ne and Kr ions in graphite. Radiat Meas. 1997;28:65-70.

[14] Liu J, Trautmann C, C.Muller, Neumann R. Graphite irradiated by swift heavy ions under grazing incidence. Nucl Instrum Methods Phys Res, Sect B. 2002;193:259-64.

[15] Novoselov KS, Geim AK, Morozov SV, Jiang D, Zhang Y, Dubonos SV, et al. Electric Field Effect in Atomically Thin Carbon Films. Science. 2004;306(5696):666-9.

[16] Novoselov KS, Jiang D, Schedin F, Booth TJ, Khotkevich VV, Morozov SV, et al. Two-dimensional atomic crystals. Proc Natl Acad Sci U S A. 2005;102(30):10451-3.

[17] Teweldebrhan D, Balandin AA. Modification of Graphene Properties due to Electron-Beam Irradiation. Appl Phys Lett. 2008;94(1):013101-3.

[18] Iqbal MZ, Kelekci O, Iqbal MW, Eom J. The structural and electrical evolution of chemical vapor deposition grown graphene by electron beam irradiation induced disorder. Carbon. 2013;59:366-71.

[19] Compagnini G, Giannazzo F, Sonde S, Raineri V, Rimini E. Ion irradiation and defect formation in single layer 
graphene. Carbon. 2009;47(14):3201-7.

[20] Chen JH, Cullen W, Jang C, Fuhrer M, Williams E. Defect Scattering in Graphene. Phys Rev Lett. 2009;102(23):236805.

[21] Lucchese MM, Stavale F, Ferreira EHM, Vilani C, Moutinho MVO, Capaz RB, et al. Quantifying ion-induced defects and Raman relaxation length in graphene. Carbon. 2010;48(5):1592-7.

[22] Jorio A, Lucchese MM, Stavale F, Ferreira EH, Moutinho MV, Capaz RB, et al. Raman study of ion-induced defects in N-layer graphene. Journal of physics Condensed matter : an Institute of Physics journal. 2010;22(33):334204.

[23] Martins Ferreira EH, Moutinho MVO, Stavale F, Lucchese MM, Capaz RB, Achete CA, et al. Evolution of the Raman spectra from single-, few-, and many-layer graphene with increasing disorder. Phys Rev B. 2010;82(12):125429.

[24] Giro R, Archanjo BS, Martins Ferreira EH, Capaz RB, Jorio A, Achete CA. Quantifying defects in N-layer graphene via a phenomenological model of Raman spectroscopy. Nucl Instrum Methods Phys Res, Sect B. 2014;319:71-4.

[25] Novoselov KS, Geim AK, Morozov SV, Jiang D, Zhang Y, Dubonos SV, et al. Electric field effect in atomically thin carbon films. Science. 2004;306(5696):666-9

[26] Novoselov KS, Jiang D, Schedin F, Booth TJ, Khotkevich VV, Morozov SV, et al. Two-dimensional atomic crystals. Proc Natl Acad Sci U S A. 2005;102(30):10451-3.

[27] Ziegler JF, Ziegler MD, Biersack JP. SRIM - The stopping and range of ions in matter (2010). Nucl Instrum Methods Phys Res, Sect B. 2010;268(11-12):1818-23.

[28] Ziegler J, Biersack J, Ziegler M, Marwick D, Cuomo G, Porter W, et al. SRIM-2013 code.

[29] Nakamura K, Kitajima M. Ion-irradiation effects on the phonon correlation length of graphite studied by Raman spectroscopy. Phys Rev B. 1992;45(1):78-82.

[30] Theodosiou A, Carley AF, Taylor SH. Ion-induced damage in graphite: A Raman study. J Nucl Mater. 2010;403(1-3):108-12.

[31] Zhai PF, Liu J, Duan JL, Chang HL, Zeng J, Hou MD, et al. Velocity effect of swift heavy ions in graphite studied by Raman spectroscopy. Nucl Instrum Methods Phys Res, Sect B. 2011;269(18):2035-9.

[32] Ferrari AC, Robertson J. Interpretation of Raman spectra of disordered and amorphous carbon. Phys Rev B. 2000;61(20):14095-107.

[33] Castiglioni C, Negri F, Rigolio M, Zerbi G. Raman activation in disordered graphites of the A1 symmetry forbidden k0 phonon: The origin of the D line. The Journal of Chemical Physics. 2001;115(8):3769.

[34] Reich S, Thomsen C. Raman spectroscopy of graphite. Philos T Roy Soc A. 2004;362(1824):2271-88.

[35] Eckmann A, Felten A, Mishchenko A, Britnell L, Krupke R, Novoselov KS, et al. Probing the Nature of Defects in Graphene by Raman spectroscopy. Nano Lett. 2012;12(8):3925-30.

[36] Gouadec G, Colomban P. Raman Spectroscopy of nanomaterials: How spectra relate to disorder, particle size and mechanical properties. Prog Cryst Growth Ch. 2007;53(1):1-56.

[37] Katumba G, Mwakikunga BW, Mothibinyane TR. FTIR and Raman Spectroscopy of Carbon Nanoparticles in SiO2, ZnO and NiO Matrices. Nanoscale Research Letters. 2008;3(11):421-6.

[38] Zhai P-F, Liu J, Zeng J, Yao H-J, Duan J-L, Hou M-D, et al. Raman spectrum study of graphite irradiated by swift heavy ions. Chinese Physics B. 2014;23(12):126105.

[39] Zeng J, Yao HJ, Zhang SX, Zhai PF, Duan JL, Sun YM, et al. Swift heavy ions induced irradiation effects in monolayer graphene and highly oriented pyrolytic graphite. Nucl Instrum Methods Phys Res, Sect B. 2014;330:18-23.

[40] Watanabe H, Takahashi K, Iwaki M. Structural characterization of ion implanted pyrolytic graphite. Nucl Instrum 
Methods Phys Res, Sect B. 2007;257(1-2):549-53.

[41] Wang TS, Ding JJ, Cheng R, Peng HB, Lu X, Zhao YT. Diamond-Like Carbon produced by highly charged ions impact on highly oriented pyrolytic graphite. Nucl Instrum Methods Phys Res, Sect B. 2012;272:15-7.

[42] F.Tuinstra, Koenig JL. Raman Spectrum of Graphite. The Journal of Chemical Physics. 1970;53(3):1126-30.

[43] Knight DS, White WB. Characterization of diamond films by Raman spectroscopy. J Mater Res. 1989;4:385.

[44] Cançado LG, Takai K, Enoki T, Endo M, Kim YA, Mizusaki H, et al. Measuring the degree of stacking order in graphite by Raman spectroscopy. Carbon. 2008;46(2):272-5.

[45] Cançado LG, Takai K, Enoki T, Endo M, Kim YA, Mizusaki H, et al. General equation for the determination of the crystallite size L[sub a] of nanographite by Raman spectroscopy. Appl Phys Lett. 2006;88(16):163106.

[46] Cançado LG, Jorio A, Ferreira EHM, Stavale F, Achete CA, Capaz RB, et al. Quantifying defects in graphene via Raman spectroscopy at different excitation energies. Nano Lett. 2011;11(8):3190-6.

[47] Zeng J, Zhai PF, Liu J, Yao HJ, Duan JL, Hou MD, et al. Production of sp3 hybridization by swift heavy ion irradiation of HOPG. Nucl Instrum Methods Phys Res, Sect B. 2013;307:562-5.

[48] Liu J, Hou MD, Liu CL, Wang ZG, Jin YF, Zhai PJ, et al. Tracks of high energy heavy ions in HOPG studied with scanning tunneling microscopy. Nucl Instrum Methods Phys Res, Sect B. 1998;146:356-61.

[49] Ishikawa N, Chimi Y, Michikami O, Hashimoto T, Kambara T, Neumann R, et al. Ion-velocity dependence of high-density electronic excitation effects in oxide superconductors. Nucl Instrum Methods Phys Res, Sect B. 2005;230(1-4):136-41.

[50] Meftah A, Brisard F, Costantini J, Hage-Ali M, Stoquert J, Studer F, et al. Swift heavy ions in magnetic insulators: A damage-cross-section velocity effect. Phys Rev B. 1993;48(2):920-5.

[51] Wang ZG, Dufour C, Cabeau B, Dural J, Fuchs G, Paumier E, et al. Velocity effect on the damage creation in metals in the electronic stopping power regime. Nucl Instrum Methods Phys Res, Sect B. 1996;107:175-80.

[52] Toulemonde M, Ramos SMM, Bernas H, Clerc C, Canut B, Chaumont J, et al. MeV gold irradiation induced damage in $\alpha$-quartz: Competition between nuclear and electronic stopping. Nucl Instrum Methods Phys Res, Sect B. 2001;178(1-4):331-6.

[53] Avrami M. Kinetics of Phase Change. I General Theory. The Journal of Chemical Physics. 1939;7(12):1103.

[54] Avrami M. Kinetics of Phase Change. II Transformation-Time Relations for Random Distribution of Nuclei. The Journal of Chemical Physics. 1940;8(2):212.

[55] Avrami M. Granulation, Phase Change, and Microstructure Kinetics of Phase Change. III. The Journal of Chemical Physics. 1941;9(2):177.

[56] Mathew S, Chan TK, Zhan D, Gopinadhan K, Barman AR, Breese MBH, et al. The effect of layer number and substrate on the stability of graphene under MeV proton beam irradiation. Carbon. 2011;49(5):1720-6.

[57] Burgdörfer J, Lerner P, Meyer F. Above-surface neutralization of highly charged ions: The classical over-the-barrier model. Phys Rev A. 1991;44(9):5674-85.

[58] Nemanich RJ, Solin SA. First- and second-order Raman scattering from finite-size crystals of graphite. Phys Rev B. 1979;20(2):392-401.

[59] Ferrari AC, Meyer JC, Scardaci V, Casiraghi C, Lazzeri M, Mauri F, et al. Raman Spectrum of Graphene and Graphene Layers. Phys Rev Lett. 2006;97(18):187401.

[60] Zeng J, Liu J, Zhang S-X, Zhai P-F, Yao H-J, Duan J-L, et al. Irradiation effects of graphene and thin layer graphite induced by swift heavy ions. Chinese Physics B. 2015;24(8):086103.

[61] Lehtinen O, Kotakoski J, Krasheninnikov AV, Tolvanen A, Nordlund K, Keinonen J. Effects of ion bombardment on a two-dimensional target: Atomistic simulations of graphene irradiation. Phys Rev B. 2010;81(15):153401.

[62] Krasheninnikov A, Nordlund K, Sirviö M, Salonen E, Keinonen J. Formation of ion-irradiation-induced atomic-scale defects on walls of carbon nanotubes. Phys Rev B. 2001;63(24):2405405. 
[63] Ouyang M, Huang JL, Cheung CL, Lieber CM. Atomically resolved single-walled carbon nanotube intramolecular junctions. Science. 2001;291(5501):97-100.

3 [64] Hashimoto A, Suenaga K, Gloter A, Urita K, Iijima S. Direct evidence for atomic defects in graphene layers. Nature. 4 2004;430:870-3.

5 [65] Meyer JC, Eder F, Kurasch S, Skakalova V, Kotakoski J, Park HJ, et al. Accurate measurement of electron beam induced displacement cross sections for single-layer graphene. Phys Rev Lett. 2012;108(19):196102.

[66] Lazzeri M, Attaccalite C, Wirtz L, Mauri F. Impact of the electron-electron correlation on phonon dispersion: Failure of LDA and GGA DFT functionals in graphene and graphite. Phys Rev B. 2008;78(8):081406.

[67] Arnau A, Aumayr F, Echenique PM, Grether M, Heiland W, Limburg J, et al. Interaction of slow multicharged ions with solid surfaces. Surf Sci Rep. 1997;27(4-6):113-5, 7-239.

[68] Gillaspy JD. Highly charged ions. Journal of Physics B: Atomic, Molecular and Optical Physics. 2001;34:R93-R130.

[69] E.Asari. An effect of the extended cascade on the Raman spectra of ion-irradiated graphite. Carbon. 2000;38(13):1857-61.

[70] Zeng J, Yao H, Mo D, Duan J, Liu J, Cao D, et al. Ion current rectification effect of porous graphene membrane. physica status solidi (c). 2014:1-5. 